\title{
Effect of Oligothiophene as a Buffer Layer on the OLED of the Convergence Relation with Varying Thickness
}

\author{
Ho Shik Lee, Professor, Department of Health Administration, DongShin University, Korea, 58245 Naju \\ Hyun Woo Jeong, Professor, College of Korean Medicine, DongShin University, Korea, 58245 Naju \\ *Jae Hwan Kwon, Professor, Graduate School of Education, DongShin University, Korea, 58245 Naju, \\ jhkwon@dsu.ac.kr \\ *Corresponding Author
}

Abstract. Background/Objectives: In this article the buffer materials, $\alpha-6 \mathrm{~T}$ and $\alpha-7 \mathrm{~T}$ are used to study the interface of metla/organic in OLEDs. It has been observed that the electrical, light emitting and efficiency properties are strongly depend on the thickness of the buffer layer with $60 \mathrm{~nm}$ of $\alpha-6 \mathrm{~T}$ and $3 \mathrm{~nm}$ of $\alpha$-7T the mostly improved properties were observed.

Methods/Statistical analysis: The physical interaction of molecular at the metal/organic interface has been investigated by contact angle hysteresis. The surface energy of oligothiophene layer on ITO electrode varies within $10 \mathrm{~mJ} / \mathrm{cm}^{2}$. The polar component of surface energy of organic materials(HTL) on buffer layer materials was observed to be independent on the thickness of buffer layer with the value of zero. The strong electrostatic force of the metallic surface may lead the properties of organic materials to be different from that of natural state. However the buffer layer may contribute to the continuously layered similar energy state that increase the efficiency of hole injection.

Findings: It has been observed that the electrical properties, light emitting and efficiency properties are strongly depend on the thickness of the buffer layer with $60 \mathrm{~nm}$ of $\alpha-6 \mathrm{~T}$ and $3 \mathrm{~nm}$ of $\alpha-7 \mathrm{~T}$ the mostly improved properties were observed. The lowered energy barrier, about $0.2 \sim 0.4 \mathrm{eV}$, for hole injection at the interface of metal/buffer layer and the improved surface morphology of hole injection surface may contribute to the improvements. It was expected that the increase of the absolute number of holes injected might increase the light emitting efficiency and it was observed.

Improvements/Applications: As a tool to investigate such as phenomena, the use of buffer materials gives advantages. The buffer materials normally show stable chemical and physical properties, it could be avoided the destruction of original electronic state during the measurement. The use of buffer materials and the understandings of interface phenomena could offer many applicable products to our common life.

Keywords: Buffer layer, $\alpha-6 \mathrm{~T}, \alpha-7 \mathrm{~T}$, interface, oligothiophene, hole injection

Received: 08.12.2020 Accepted: 12.01.2021 Published: 07.02.2021

\section{Introduction}

Developing and developing ways to display information is one of the efforts that humans have consistently made. Rapid development of electrical energy is possible is the conversion of electrical energy into light energy and use it as a method of information display. The same phenomenon can be observed using organic materials, but the problem raised during the initial development phase is low 
energy conversion efficiency [1-4].

Organic light emitting diodes (OLEDs) have limitations in their use of minerals (metal or semiconductor) as suppliers of electrical energy. The disadvantage of not being able to exclude minerals lies in the energy barriers present on the interface of inorganic (metal or semiconductor)/organic materials. Energy barrier is known to have a significant impact on the efficiency of the overall device drive and also play an important role in the stability of the device [5-7].

As one of the information display elements using organic materials, OLEDs are subject to many studies due to their necessity and utility. Also, the OLEDs are capable of operating at direct current low voltages, unlike those operated under alternating current high voltages.

This increase in efficiency is due to the availability of effective perforation and electronic transmission in the interface between organic matter/electrode. Organic materials applied to the OLEDs are manufactured in thin film form within hundreds of $\mathrm{nm}$, and it is an important point of view whether they can exist in a stable solid ultra thin film state. If electrical luminescence was viewed as the emission of electromagnetic waves from solid state materials by external electric fields, the process can be divided into the injection of electrons or holes and the transport electron by carriers and the luminescence stage after recombination.

In this paper, the interface of metal/organic materials in OLED is studied using buffer materials $\alpha-6 \mathrm{~T}$ and $\alpha-7$ T. The characteristics of electricity, luminescence and efficiency were observed to vary greatly depending on the thickness of the buffer layer of $\alpha-6 \mathrm{~T} 60 \mathrm{~nm}$ and $\alpha-7 \mathrm{~T} 3 \mathrm{~nm}$. Low energy barrier of about 0.2 to $0.4 \mathrm{eV}$ for hole injection in the interface of the metal/buffer layer and improved surface morphology of hole injection surface can cause improvement. It was observed that increasing the number of absolute holes injected will increase luminous efficiency. 


\section{Experimntals}

\subsection{Materials}

Polyvinylcarbazol(PVK)(Kanto Chemical Co., INC), N, N'-diphenyl-N, N'm-tolyl(m-tolyl)-benzine(TPD)(TCI) was used as a hole transfer material in this study. 8-hydroxyquinoline, aluminum salt $\left(\mathrm{Alq}_{3}\right)$ as an electronic transfer material and lighting materials (Aldrich), 2-(4-biphenyl)-5-(4-tert-butyl)-1,3,4-oxadiazole)(PBD),also, 1,4,4-tetraphenyl-1,3-butadene)(TPB) were used as an electron transfer materials. Also, $\alpha$-sexythiophene $(\alpha-6 \mathrm{~T})$ and $\alpha$-septithiophene $(\alpha-7 \mathrm{~T})$ as buffer material was used. $\mathrm{Alq}_{3}$ and TPD films were vacuum deposited and PVK film was made by spin-coating method. ITO (Indium-tin-oxide, Samsung Corning Co. LTD) was used an anode electrode and LiF was used a buffer layer and $\mathrm{Al}$ as a cathode electrode was used in this study. Figure 1 shows the molecular structure of an Alq3, TPD, PVK, $\alpha-6 \mathrm{~T}$, and $\alpha-7 \mathrm{~T}$ used in this study.

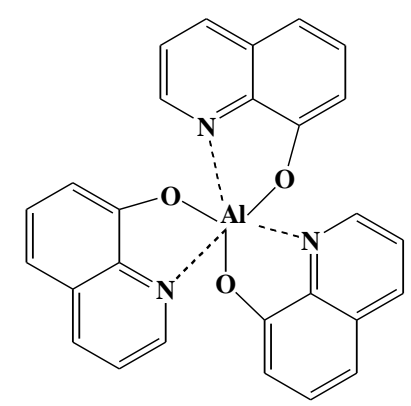

(a) $\mathrm{Alq}_{3}$

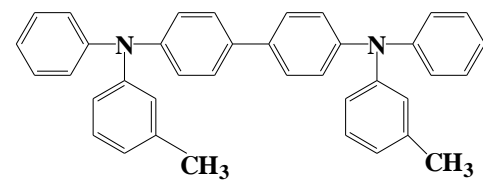

(b) TPD

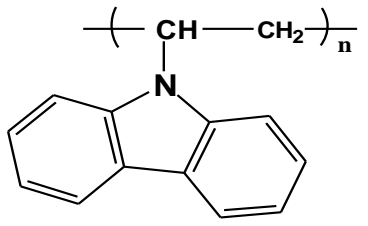

(c) PVK

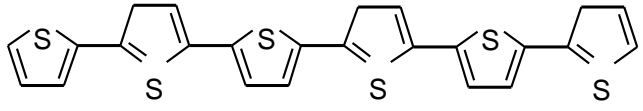

(d) $\alpha-6 \mathrm{~T}$

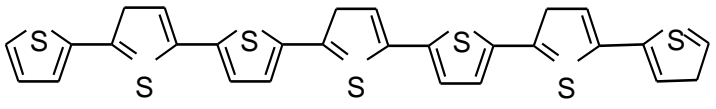

(e) $\alpha-7 \mathrm{~T}$

Fiurure 1. Molecular structure.

\subsection{Thin films preparation}

Organic thin films were holy using vacuum heat deposition method, and the thickness of thin film was confirmed by QCM (Quartz crystal microbalance) and elliptical analysis method. Photoluminescence (PL) peak in solid thin film conditions of $\mathrm{Alq}_{3}$ and rubrene is known to be $510 \mathrm{~nm}$ and $550 \mathrm{~nm}$, respectively [8-10]. Also, PVK was fabricated onto pre-cleaned ITO by spin-coating method. The ITO glass was cleaned by sonicated with chloroform for $20 \mathrm{~min}$ at $50^{\circ} \mathrm{C}$ and heated at $80^{\circ} \mathrm{Cfor} 1 \mathrm{~h}$ with distilled deionized water. Table 1 shows the thin film fabrication condition of an organic materials used in this study. 
Table 1. Thin film fabrication condition.

\begin{tabular}{|c|c|c|c|c|c|c|}
\hline & TPD & Alq 3 & $a-6 \mathrm{~T}$ & $\alpha-7 \mathrm{~T}$ & LiF & Al \\
\hline Molecular weight & 516.67 & 459.44 & 386.53 & 437.59 & 25.94 & 26.98 \\
\hline $\begin{array}{c}\text { Deposition } \\
\text { temperature }[\mathrm{C}]\end{array}$ & 210 & 320 & 340 & 310 & $>700$ & $>700$ \\
\hline $\begin{array}{l}\text { Deposition } \\
\text { rate }[\AA / s]\end{array}$ & $1.0 \pm 0.2$ & $0.8 \pm 0.2$ & $0.5 \pm 0.2$ & $10 . \pm 0.2$ & 0.2 & $15 \pm 2$ \\
\hline Thickness $[\mathrm{A}]$ & $10-100$ & 600 & $200-800$ & $10-200$ & 5 & 2000 \\
\hline
\end{tabular}

\section{Results and Discussion}

Figure 2(a) shows the current-voltage characteristics of the organic light emitting diodes (OLEDs) with the structure of ITO/ $\alpha-6 \mathrm{~T}(\mathrm{X} \AA) / \mathrm{TPD}(400 \AA) / \mathrm{Aql}_{3}(600 \AA) / \mathrm{Al}$ with varying thickness of the $\alpha-6 \mathrm{~T}$ using buffer layer. We were observed the applied electric field changes with spaced charge limited current depending on the thickness of the $\alpha-6 \mathrm{~T}$. The electric field was maximum at about $8 \mathrm{~V}$ at $\mathrm{X}=0 \mathrm{~nm}$ and minimum at about $6 \mathrm{~V}$ at $\mathrm{X}=40 \mathrm{~nm}$. The applied voltage was obtained the same current was lower than that not used the $\alpha-6$ T. This phenomenon can be thought to be due to the low ionization energy of $\alpha-6 \mathrm{~T}$ and the highly hole mobility.

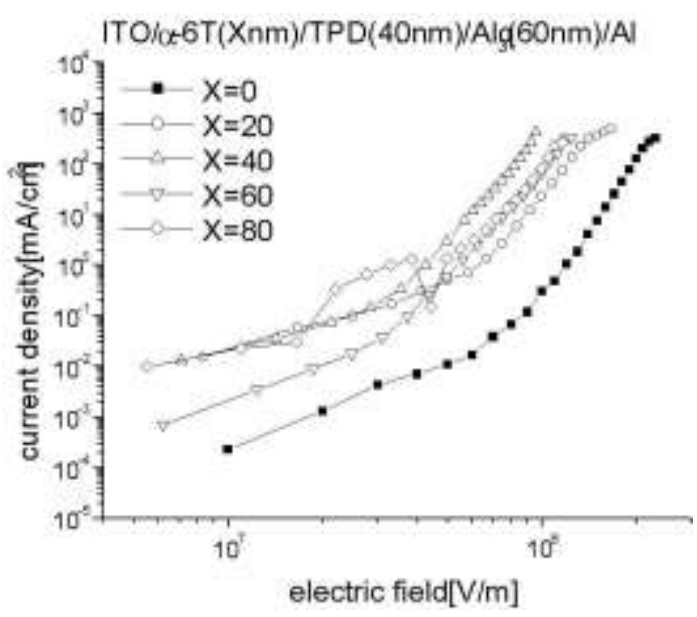

(a) Current density - electric field

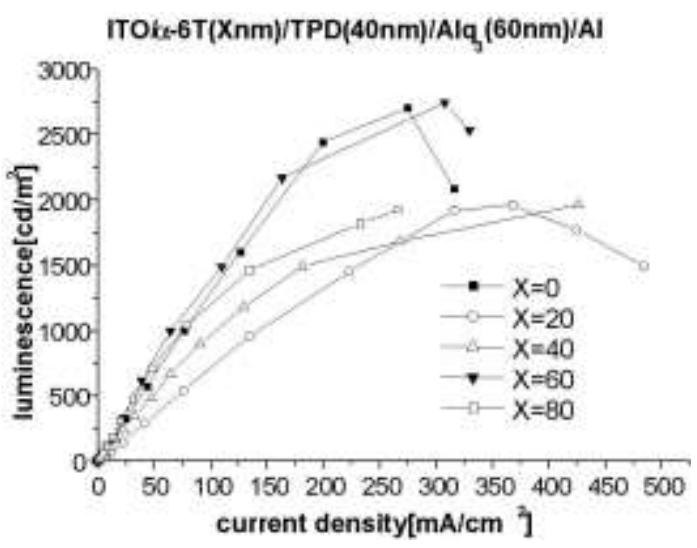

(b) Luminesence - current density 


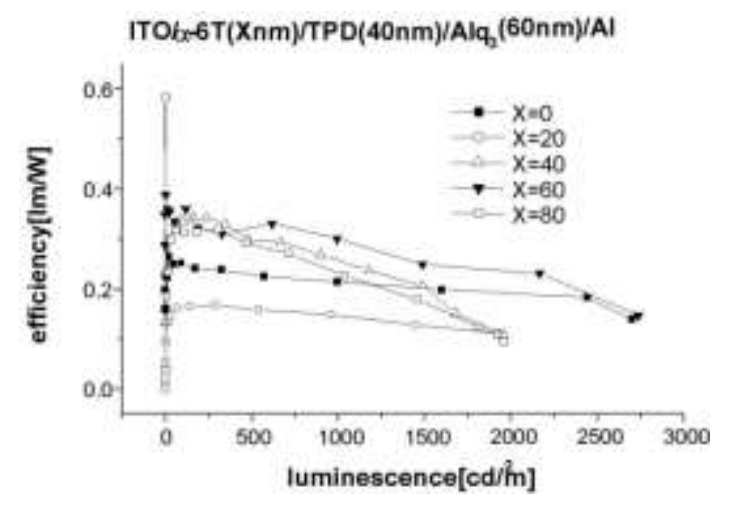

(c) Efficency - luminescence

Fiurure 2. Electical properties of the ITO/ $\left.\alpha-6 \mathrm{~T}(\mathrm{X} \AA) / \mathrm{TPD}(400 \AA)) / \mathrm{Aq}_{3}(600 \AA)\right) / \mathrm{Al}$.

Figure 2(b) shows the luminescence - current density characteristics of the OLEDs with $\alpha-6 \mathrm{~T}$. As the thickness of $\alpha-6 \mathrm{~T}$ increases, it can be seen that the luminance was increases at current density 150 $\mathrm{mA} / \mathrm{cm}^{2}$. However, the maximum luminous intensity was at about $2700 \mathrm{~cd} / \mathrm{m}^{2}$ when the thickness of $\alpha-6 \mathrm{~T}$ was $60 \mathrm{~nm}$, and at $80 \mathrm{~nm}$ the luminous intensity was reduced to about $1500 \mathrm{~cd} / \mathrm{m}^{2}$.

Figure 2(c) shows the luminous efficiency of the element with $\alpha-6 \mathrm{~T}$. We were observed luminance of $1500 \mathrm{~cd} / \mathrm{m}^{2}$ used the $\alpha-6 \mathrm{~T}$ and luminescence efficiency may be observed gradually reduced. This phenomenon, which occurs in the case of $X<60 \mathrm{~nm}$, can be thought to be caused by excess carriers injected from the $\alpha-6 \mathrm{~T}$ layer. From the Kido and Iizumi show that the imbalance in carrier injection could lead to reduced luminous efficiency [11]. When the thickness of the $\alpha-6 \mathrm{~T}$ layer was $100 \mathrm{~nm}$, the applied voltage at which the space charge limiting current began to be measured was observed to be about $3 \mathrm{~V}$. These results indicate that the $\alpha-6 \mathrm{~T}$ was a performed hole injection material in the device.

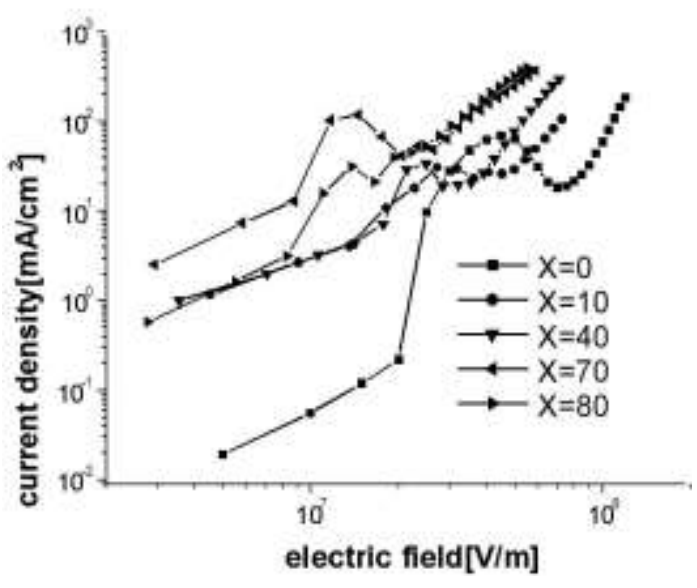

(a) Current density - electric field

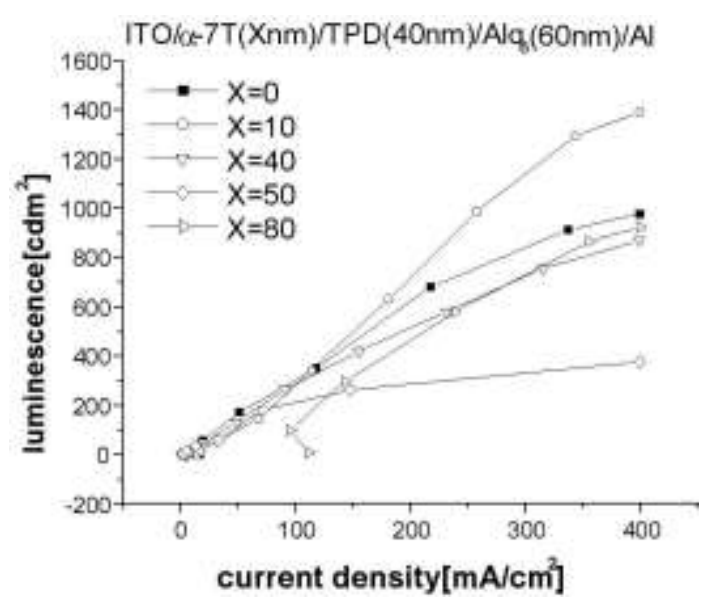

(b) Luminesence - current density 


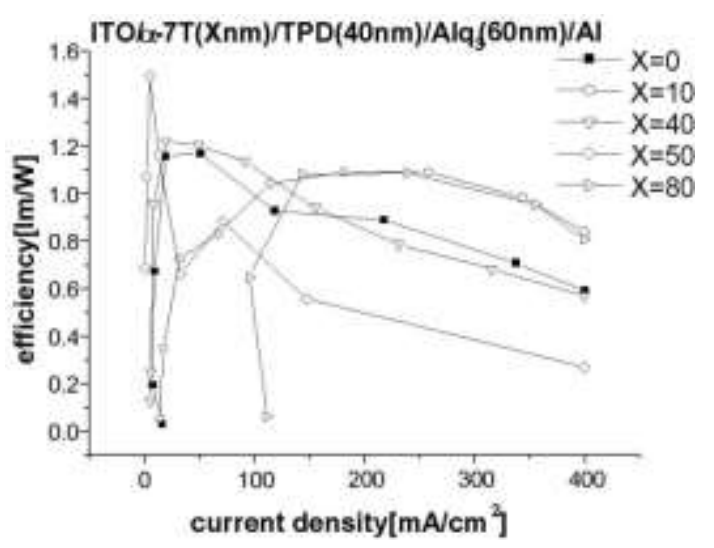

(c) Efficency - luminescence

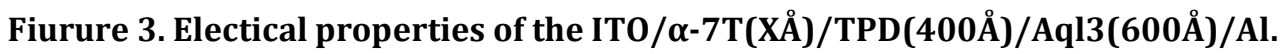

Figure 3 shows the electrical properties of the OLEDs with the structure of ITO $/ \alpha-7 \mathrm{~T}(\mathrm{Xnm}) / \mathrm{TPD}(40 \mathrm{~nm}) / \mathrm{Aql}_{3}(60 \mathrm{~nm}) / \mathrm{Al}$. The $\alpha-7 \mathrm{~T}$ was performed hole injected layer, such as $\alpha-6 \mathrm{~T}$, which is thought to be due to low ionization energy, as in the case of $\alpha-6 \mathrm{~T}$. A maximum luminance was observed at $\mathrm{X}=10 \mathrm{~nm}$ with about $1400 \mathrm{~cd} / \mathrm{m}^{2}$. The measurement of a lower luminous intensity in the case of $X=40,50$ and $80 \mathrm{~nm}$ than in the case of $X=0$ can be thought of as a reduced in efficiency due to excess hole carrier, as in the case of $\alpha-6 \mathrm{~T}$. With $\alpha-7 \mathrm{~T}$, a maximum luminous intensity of $1390 \mathrm{~cd} / \mathrm{m}^{2}$ was observed at an applied voltage of about $13 \mathrm{~V}$ when the buffer layer was formed with a thickness of $10 \mathrm{~nm}$, with a maximum luminous efficiency of $1.09 \mathrm{~lm} / \mathrm{W}$ at about $985 \mathrm{~cd} / \mathrm{m}^{2}$.

We made the single type OLEDs using conductive polymer PVK, and we were observe the change in the form of a hole injection by the buffer layer. The PVK material was as a hole transfer material, a PBD as an electron transfer material and TPB as a lighting materials were used of the OLEDs. In the case of organic electro-luminescent devices in ITO/PVK:TPB:PBD/Al structures as shown in Figure 4(a), the hole injection at the ITO/PVK interface is well matched in behavior by the F-N(Fowler-Nordheim) model in Figure 4(b). The linear relationship shown in sections above or above $11 \mathrm{~V}$ indicates that the spatial charge limiting current is a descriptive model above this applied voltage. From the Figure 4(c), the F-N model is not consistent when $\alpha-6 \mathrm{~T}$ is used as a buffer layer. In this case, the energy barrier for hole injection means that most of its contribution is to the $\alpha-6 \mathrm{~T} / \mathrm{PVK}$ interface.

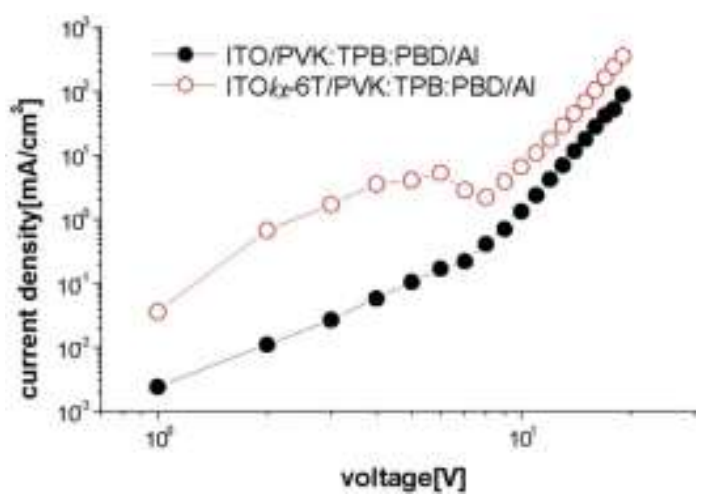


(a) Current density - voltage characteristics

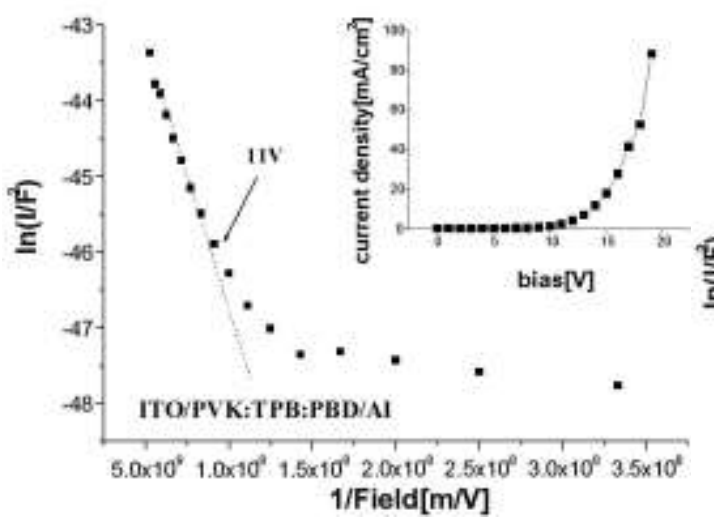

(b) Fowler-Nordheim plot

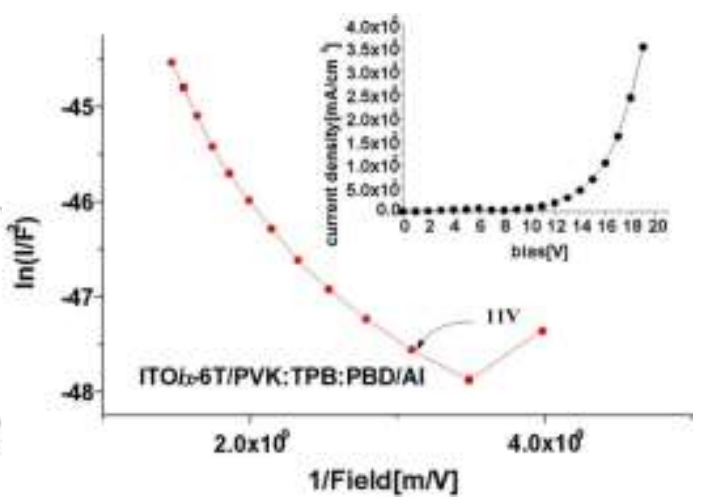

(c) ITO/PVK:TPB:PBD/Al with the

$\alpha-6 \mathrm{~T}$

Fiurure 4. Electrical properties of the OLEDs with the $\alpha-6 \mathrm{~T}$ and PVK.

Figure 5 shows the AFM images of the $\alpha-7$ T thin film with Aqua regia treatment of the ITO substrate. The surface image was observed using AFM technique to observe the surface after thin film growth of $\alpha$-7T. As the $\alpha$-7T layer grew, the filling of the ITO air gap was clearly observed and this is thought to contribute to the improvement of surface roughness.

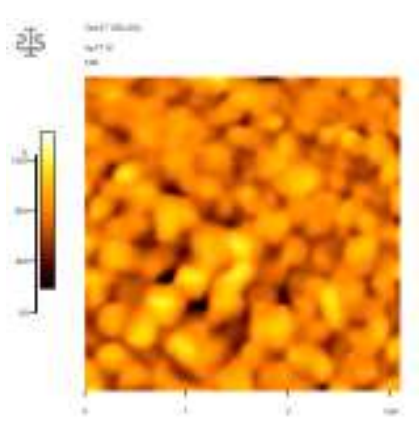

(a) $1 \mathrm{~nm}$

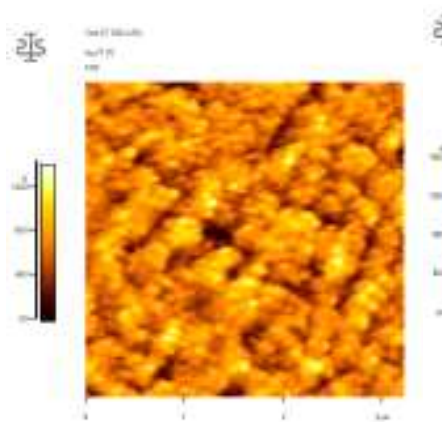

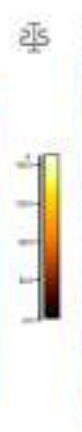
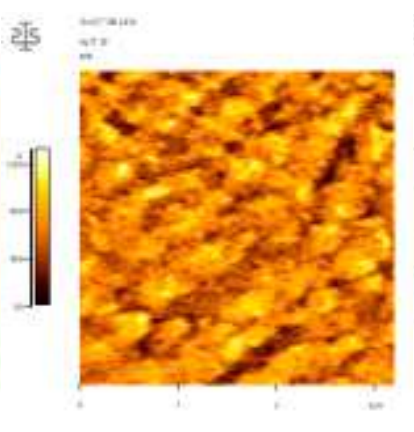

(b) $3 \mathrm{~nm}$

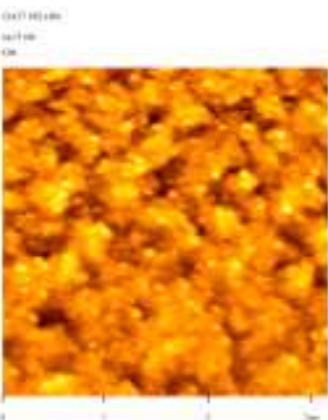

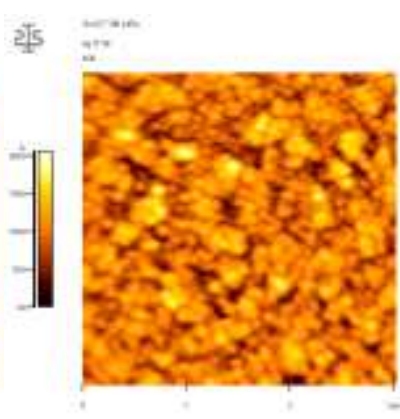

(c) $5 \mathrm{~nm}$

(d) $7 \mathrm{~nm}$

(e) $10 \mathrm{~nm}$

Figure 5. AFM images of the $\alpha-7 \mathrm{~T}$ with aquaregia treatment on the ITO.

Figure 6 shows the AFM images of the $\alpha-7$ T thin film with ultra-sonic treatment of the ITO substrate. Similar filling of air-gap was observed when the $\alpha$-7T thin film grew on the surface of the ITO 
electrode after ultrasonic cleaning. However, white dots were not observed that appeared to be the growth of the $\alpha$-7T molecule. Table 2 shows the average roughness of the $\alpha-7 \mathrm{~T}$ on the substrate with surface treatment of the ITO.

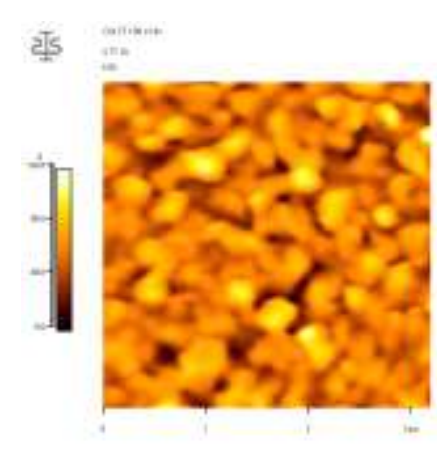

(a) $1 \mathrm{~nm}$

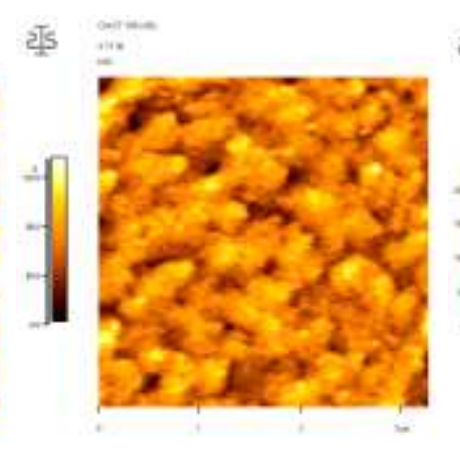

(b) $3 \mathrm{~nm}$

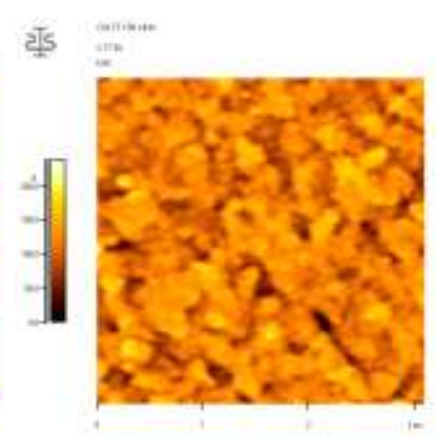

(c) $5 \mathrm{~nm}$

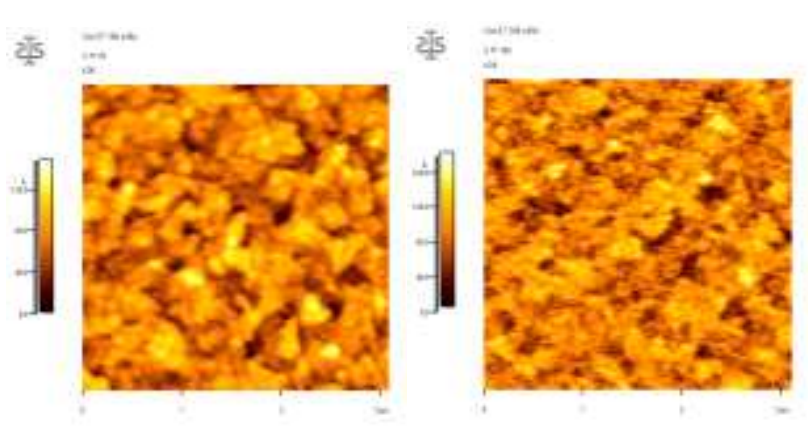
(d) $7 \mathrm{~nm}$
(e) $10 \mathrm{~nm}$

Figure 6. AFM images of the $\alpha-7$ T with ultra-sonic treatment on the ITO.

Table 2. Roughness of the $\alpha-7$ T thin film.

\begin{tabular}{|c|c|c|}
\hline Thickness of $\alpha-7 \mathrm{~T}[\mathrm{~nm}]$ & Substrate & Avg. roughness[nm] \\
\hline \multirow{2}{*}{1} & Aquaregia treatment & 1.2 \\
& Ultrasonication treatment & 1.2 \\
\hline \multirow{2}{*}{3} & Aquaregia treatment & 1.5 \\
& Ultrasonication treatment & 1.4 \\
\hline \multirow{2}{*}{5} & Aquaregia treatment & 2.2 \\
& Ultrasonication treatment & 2.0 \\
\hline \multirow{2}{*}{7} & Aquaregia treatment & 1.6 \\
& Ultrasonication treatment & 1.6 \\
\hline \multirow{2}{*}{10} & Aquaregia treatment & 1.7 \\
& Ultrasonication treatment & 1.9 \\
\hline
\end{tabular}

\section{Conclusion}

We were observed the electrons distribution of the organic/organic and organic/inorganic interface. The increase in efficiency caused by the change in the thickness of the buffer layer was observed. Also, the 
surface characteristics of the ITO/organic interface were studied by AFM technique. We were observed the current density and luminescence efficiency were increased of the OLEDs using the buffer layer with the oligothiophene. With $\alpha-7 \mathrm{~T}$, a maximum luminous intensity of $1390 \mathrm{~cd} / \mathrm{m}^{2}$ was observed at an applied voltage about $13 \mathrm{~V}$ when the buffer layer was formed with a thickness of $10 \mathrm{~nm}$, with a maximum luminous efficiency of $1.09 \mathrm{~lm} / \mathrm{W}$ at about $985 \mathrm{~cd} / \mathrm{m}^{2}$. The reason why $\alpha-6 \mathrm{~T}$ has a relatively high luminous intensity but a low value in luminous efficiency can be attributed to the increase in the relative applied voltage due to the high energy barriers present in the interface with the ITO electrode. The luminous intensity and luminous efficiency of the OLEDs are greatly influenced by the energy barriers on the ITO/organic interface and can induce a rise in luminous efficiency by lowering these energy barriers. Using polymeric materials as a buffer layer, the energy barriers present in interface of ITO electrode and polymeric materials were well understood to conform to the behavior predicted by the Fowler-Nordheim tunneling theory. Using the $\alpha-6 \mathrm{~T}$ as a buffer layer, it was observed that the behavior of the hole through the energy barrier in the interface of the ITO/PVK was not very consistent with the Fowler-Nordheim tunneling theory. Also, we were not observed that linearly formed $\left[\ln \left(\mathrm{I} / \mathrm{F}^{2}\right)\right]$ and $(1 / \mathrm{F})$ using $\alpha-7 \mathrm{~T}$ but increased current density was observed. Also, the energy diagram shows that the energy barrier on the ITO $/ \mathrm{PVK}$ at about $0.5 \mathrm{eV}$ and the energy barrier on the ITO/ $\alpha-7 \mathrm{~T}$ at about $0.3 \mathrm{eV}$. We were determined that the maximum energy barrier governing the injection of the holes have shifted from the interface of the ITO/PVK to the interface of $\alpha-6 \mathrm{~T} / \mathrm{PVK}$.

The surface image was observed using AFM technique to observe the surface after thin film growth of the $\alpha-7$ T as a buffer material. As the $\alpha-7$ T layer grew, the filling of the ITO air gap was clearly observed and this is thought to contribute to the improvement of surface roughness. It can be observed that as the $\alpha-7 \mathrm{~T}$ layer grows, the white dot-shaped image increases. From this, it is judged that the ITO pole processed by the Aqua regia treatment can provide a more consistent. The $\alpha-7 \mathrm{~T}$ has very good surface roughness and this has been observed to fill the ITO's air gap well.

\section{References}

[1] I. Zschokke., H. p. Schwob. \& E. Baldinger. (1967). Recombination Radiation in Anthracene doped with tetracene, Solid State Communications 5(10), 825-828.

[2] I. G. Hill. \& A. Kahn. (1999). Organic semiconductor heterointerfaces containing bathocuproine, J. Appl . Phys. 86(8), 4515-4519.

[3] Yoshimasa. A. \& Ono. (1995). Electroluminescent displays. World Scientific, Singapore.

[4] J. Dresner. (1969). Double injection electroluminescence in anthracene. RCA Rev. 30. 322-334.

[5] C. W. Tang. \& S. A. VanSlyke. (1987). Organic electroluminescent diodes. Appl. Phys. Lett. 51(12). 913 $-915$.

[6] J. M. Caywood. (1970). Photoemisssion from Metal Contacts into Anthracene Crystals: A Critical Revie w. Mol. Cryst. Liq. Cryst. 12(1). 1-26.

[7] C. Adachi., T. Tsutsui. \& S. Saito. (1989). Electroluminescence in vacuum-deposited organic thin films. S 
pringer Proc. in Phys. Berlin. 358-361.

[8] J. Shi. \& C. W. Tang. (1997). Doped organic electroluminescent devices with improved stability. Appl. P hys. Lett. 70(13). 1665-1667.

[9] R. F. Pierret. (1996). Semiconductor Device Fundamentals. Addison Weley, Massachusetts.

[10] Dalton, E. F., Surridge, N. A., Jemigan, J. C., et al. (1990). Charge transport in electroactive polymers consisting of fixed molecular redox sites. J. Phys. Chem. 141(1). 143-157.

[11] J. Kido. \& Y. Iizumi. (1998). Fabrication of highly efficient organic electroluminescent devices. Appl. P hys, Lett. 73(19). 2721-2723. 